
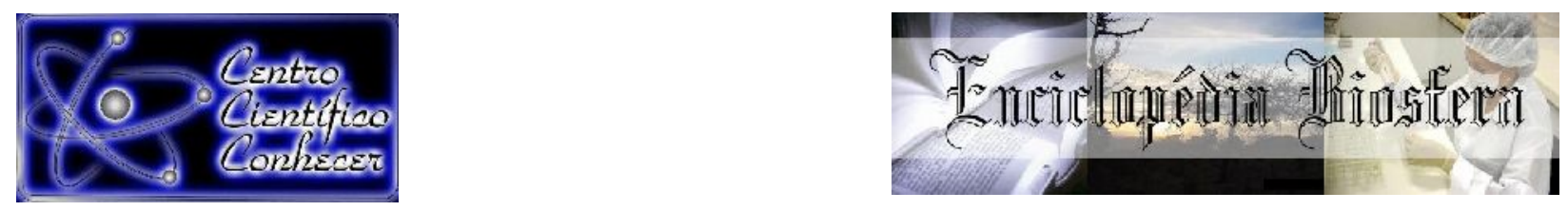

\title{
ANÁLISE RETROSPECTIVA E PROSPECTIVA DE CASOS HUMANOS DA LEISHMANIOSE TEGUMENTAR AMERICANA NO BRASIL E EM UMA ÁREA ENDÊMICA EM PERNAMBUCO
}

Elâne Rafaella Cordeiro Nunes SERAFIM ${ }^{1}$, Matheus Resende OLIVEIRA ${ }^{2}$, Igo Gonçalves dos SANTOS ${ }^{2}$, Manuel Benicio OLIVEIRA NETO², Victor Fernando Santana LIMA ${ }^{3}$

${ }^{1}$ Técnica da Vigilância em Saúde, Cupira-PE, Brasil.

${ }^{2}$ Graduando em Medicina Veterinária na UFS, Nossa Senhora da Glória - SE, Brasil

${ }^{3}$ Professor Doutor do curso de Medicina Veterinária da Universidade Federal de Sergipe, Nossa Senhora da Glória- SE, Brasil. *E-mail: victor.fslima@gmail.com

Recebido em: 15/08/2020 - Aprovado em: 15/09/2020 - Publicado em: 30/09/2020 DOI: 10.18677/EnciBio_2020C6

\begin{abstract}
A Leishmaniose Tegumentar Americana é uma zoonose negligenciada, causada por protozoários do gênero Leishmania, considerada um grande problema de saúde pública em diferentes países. Informações sobre os aspectos da cadeia epidemiológica e população afetada por essa doença é importante para estabelecer medidas profiláticas. Desse modo, o objetivo deste estudo foi analisar retrospectivamente e prospectivamente a dinâmica dos casos de Leishmaniose Tegumentar Americana em humanos no âmbito nacional notificados no período de 2009 a 2013. Os dados foram obtidos por meio do banco de dados do Sistema de Informação de Agravos de Notificação, do Ministério da Saúde. Na análise estatística os casos foram agrupados por Regiões, Estados e a IV Região Administrativa de Saúde de Pernambuco. Utilizou-se o programa EpilnfoTM 7 para execução dos cálculos, e o software Google Earth® e Quantum GIS 2.8.1., para elaboração e confecção dos mapas. Observou-se que casos de LTA foram registrados em todas as regiões do País, sendo o Norte e Centro-oeste as regiões com maior prevalência. Nas regiões Norte, Nordeste e Centro-Oeste a prevalência oscilou. No Sul e Sudeste a LTA ocorre de forma constante. Houve variação na prevalência da enfermidade por unidade federativa. Em Pernambuco observou-se uma tendência de decréscimo. A diferença observada na prevalência da LTA no País ocorre devido às variações das condições climáticas regionais, influenciando nos índices reprodutivos dos flebotomíneos, potencializando a transmissão da enfermidade. As estratégias de prevenção da LTA devem ser reavaliadas e incluir mecanismos que avaliem todos os aspectos envolvidos na cadeia epidemiológica em cada unidade federativa.
\end{abstract}

RESUMO

PALAVRAS-CHAVE: Humanos, Leishmaniose, Zoonose. 


\title{
RETROSPECTIVE AND PROSPECTIVE ANALYSIS OF HUMAN CASES OF AMERICAN CUTANEOUS LEISHMANIASIS IN BRAZIL AND IN ONE ENDEMIC AREA IN PERNAMBUCO
}

\begin{abstract}
American Tegumentary Leishmaniasis is a neglected zoonosis, caused by protozoa of the genus Leishmania, considered a major public health problem in different countries. Information on aspects of the epidemiological chain and population affected by this disease is important for establishing prophylactic measures. Thus, the objective of this study was to retrospectively and prospectively analyze the dynamics of cases of American Tegumentary Leishmaniasis in humans at the national level notified in the period from 2009 to 2013. The data were obtained through the database of the Health Information System of Notification, from the Ministry of Health. In the statistical analysis, the cases were grouped by Regions, States and the IV Administrative Health Region of Pernambuco. The EpilnfoTM 7 program was used to perform the calculations, and the Google Earth® and Quantum GIS 2.8.1. Software were used to prepare and make the maps. It was observed that cases of ATL were recorded in all regions of the country, with the North and CenterWest being the regions with the highest prevalence. In the North, Northeast and Midwest regions the prevalence fluctuated. In the South and Southeast, ATL occurs constantly. There was variation in the prevalence of the disease by federative unit. In Pernambuco there was a downward trend. The difference observed in the prevalence of ATL in the country is due to variations in regional climatic conditions, influencing the reproductive rates of sandflies, increasing the transmission of the disease. The ATL prevention strategies must be reevaluated and include mechanisms that assess all aspects involved in the epidemiological chain in each federative unit.
\end{abstract}

KEYWORDS: Humans, Leishmaniasis, Zoonosis.

\section{INTRODUÇÃO}

A Leishmaniose Tegumentar Americana (LTA) é considerada uma doença negligenciada (GALGAMUWA et al., 2018), infecciosa, endêmica, não contagiosa, zoonótica, causada por mais de cinco espécies de protozoários do gênero Leishmania (BRASIL, 2010; CAETANO et al., 2019), tida como as mais importantes relatadas no Brasil, a Leishmania (V.) braziliensi principal responsável pela origem e manifestação clínica clássica da doença, L. amazonenses e L. (V.) guyanensis (FERNANDES et al., 2016; VASCONCELOS et. al., 2018), as quais provocam lesões ulcerativas destrutivas $e$ incapacitantes, com grande comprometimento psicológico, refletindo no âmbito social e econômico do indivíduo (GONTIJO; CARVALHO, 2003; BRASIL, 2007).

Sabe-se que é uma zoonose de ocorrência cosmopolita, considerada um grande problema de saúde pública em mais de 88 países, acometendo anualmente cerca de 700.000 a um milhão de pessoas, totalizando em torno de 26.000 a 65 mil mortes em todo o mundo (BRASIL, 2007; OMS, 2020), sendo relatada em todas as regiões do Brasil, sob diferentes perfis epidemiológicos (BRASIL, 2019; BRITO et al., 2012).

A LTA apresenta distintos e complexos padrões de transmissão, em função da diversidade de espécies do parasita, hospedeiros, reservatórios e vetores (CURTI et al., 2009; FERREIRA et al., 2015). A dinâmica de padrão de transmissão, diferenciada em nível regional e local quanto aos aspectos geográficos específicos relacionados aos parasitos, vetores, ecossistemas e processos sociais de produção 
e uso do solo (BARCELLOS et al., 2009; NEGRÃO; FERREIRA, 2014). Em virtude dessas características epidemiológicas, as estratégias de controle devem ser flexíveis, distintas e adequadas para cada região ou foco em particular (BRASIL, 2010).

Uma vez que a LTA tem sido relatada em diferentes territórios, no Brasil a região Norte demonstra o maior coeficiente, 46,4 casos por 100.000 habitantes, acompanhada das regiões Centro-Oeste com 17,2 casos por 10.000 habitantes e Nordeste com oito casos por 100.000 habitantes (BRASIL, 2019). Inicialmente apontada como uma doença restrita ao ambiente rural, hoje em dia apresenta-se em constante crescimento no ambiente periurbano (ARAUJO et al., 2015).

Apesar de não ser completamente compreendido o processo de urbanização no Brasil, dentre os principais fatores que contribuem para a prevalência da doença, destaca-se o cão, revelando a sua importância como o principal reservatório doméstico em áreas urbanas, atualmente uma das características mais importantes do perfil epidemiológico da população infectada (NEGRÃO; FERREIRA, 2009; DANTAS-TORRES et al., 2017). Cabe enfatizar as relações com seus vetores, flebotomíneos (Ordem Díptera; Família Psychodidae; Sub-Família Phlebotominae) do gênero Lutzomyia, insetos fêmeas que transmitem a doença durante o repasto sanguíneo, sendo os mais comuns $L$. flaviscutellata, L. whitmani, L. umbratilis, $L$. intermedia, $L$. wellcome $e, L$. migonei em que podem ser biologicamente compatíveis, atraídos espontaneamente ou influenciados pelas intervenções humanas na natureza, culminando em ciclos distintos de transmissão (BRASIL, 2010; SHIMABUKURO; GALATI, 2011; SANTOS et al., 2018).

A obtenção de informações sobre os aspectos implicados na cadeia epidemiológica da LTA e a população afetada é de fundamental importância para o estabelecimento de medidas profiláticas (GONTIJO; CARVALHO, 2003). Desse modo, o objetivo deste estudo foi analisar retrospectivamente e prospectivamente a dinâmica dos casos de Leishmaniose Tegumentar Americana em humanos no âmbito nacional notificados no período de 2009 a 2013.

\section{MATERIAL E MÉTODOS}

Nesse estudo foram coletados no período de março a abril de 2017, os dados por meio do banco de dados do Sistema de Informação de Agravos de Notificação (SINAN), do Ministério da Saúde, referentes a casos de LTA em humanos notificados no Brasil, no período de 2009 a 2013.

As variáveis avaliadas foram: unidade federativa de residência, zona de residência, sexo e faixa etária. Informações sobre nome e/ou endereços dos pacientes não são acessadas e/ou disponibilizadas, a fim de manter a identidade dos indivíduos preservada.

Para análise estatística os casos foram agrupados por Regiões, Estados e a IV Região Administrativa de Saúde de Pernambuco. Foram utilizados dados do censo demográfico relacionados à área urbana e rural em território nacional de 2010, índice de desenvolvimento humano e produto interno bruto, per capita, dos municípios, disponibilizados pelo Instituto Brasileiro de Geografia e Estatística (IBGE), para cálculo de prevalência e regressão linear.

O programa EpilnfoTM 7 foi utilizado para a execução dos cálculos estatísticos e o nível de significância adotado foi de 5,0\%. Para confirmação das coordenadas geográficas utilizou-se o software Google Earth® e a elaboração e confecção dos mapas deu-se por meio do software Quantum GIS 2.8.1. 


\section{RESULTADOS E DISCUSSÃO}

Os casos de LTA foram registrados em todas das regiões do Brasil, sendo o Norte e Centro-oeste as regiões com maior prevalência, conforme visualizado na figura 1, sendo a prevalência nacional de $6,49(113.673 / 174.886 .771)$ casos para cada 10.000 habitantes.

FIGURA 1 - Prevalência de Leishmaniose Tegumentar Americana em humanos para 10.000 habitantes nas diferentes regiões do Brasil, no período de 2009 a 2013.

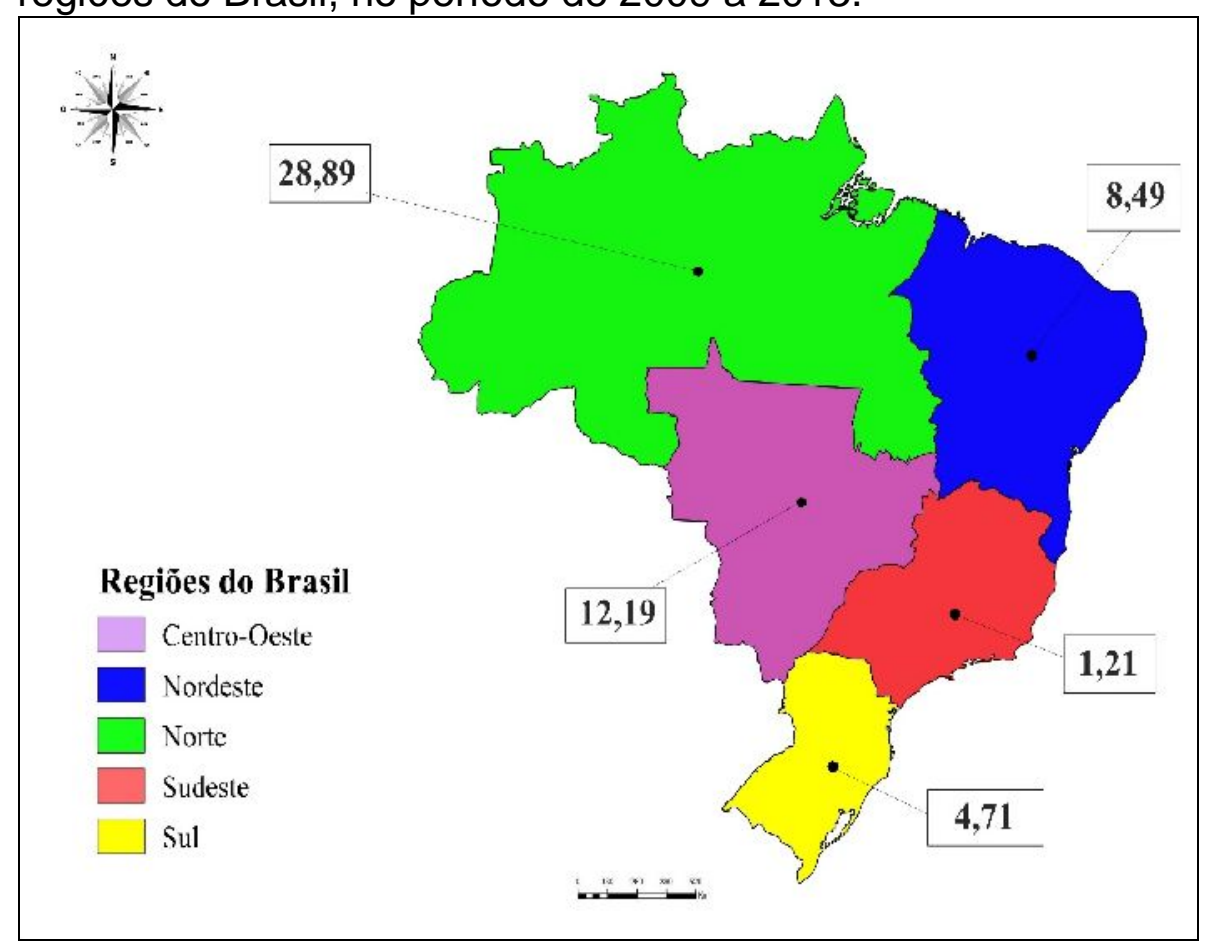

Fonte: Adaptado do SINAN (2013)

Houve variação na prevalência da enfermidade por unidade federativa, sendo observado no Maranhão o maior número de casos, seguido por Roraima e Acre, como pode ser visualizado na Figura 2.

A proporção de pacientes acometidos com LTA a cada 10.000 habitantes nas regiões Norte, Nordeste e Centro-Oeste oscilou durante o período avaliado, com tendência a aumento e em seguida decréscimo. No Sul e Sudeste a enfermidade ocorre de forma constante, no entanto em menor proporção que as outras regiões, conforme indica a Figura 3.

O Estado de Pernambuco destaca-se como área endêmica da região Nordeste, no qual no período avaliado observou-se uma tendência de decréscimo da prevalência da LTA. Embora, em uma área endêmica específica inserida na mesorregião do Agreste Pernambucano, os valores permaneceram constantes na maior parte do período avaliado com ocorrência de pico epidêmico, no início do ano de 2011, assemelhando-se ao perfil da prevalência nacional. 
FIGURA 2 - Prevalência de Leishmaniose Tegumentar Americana em humanos para 10.000 habitantes nas Unidades Federativa do país, no período de 2009 a 2013.

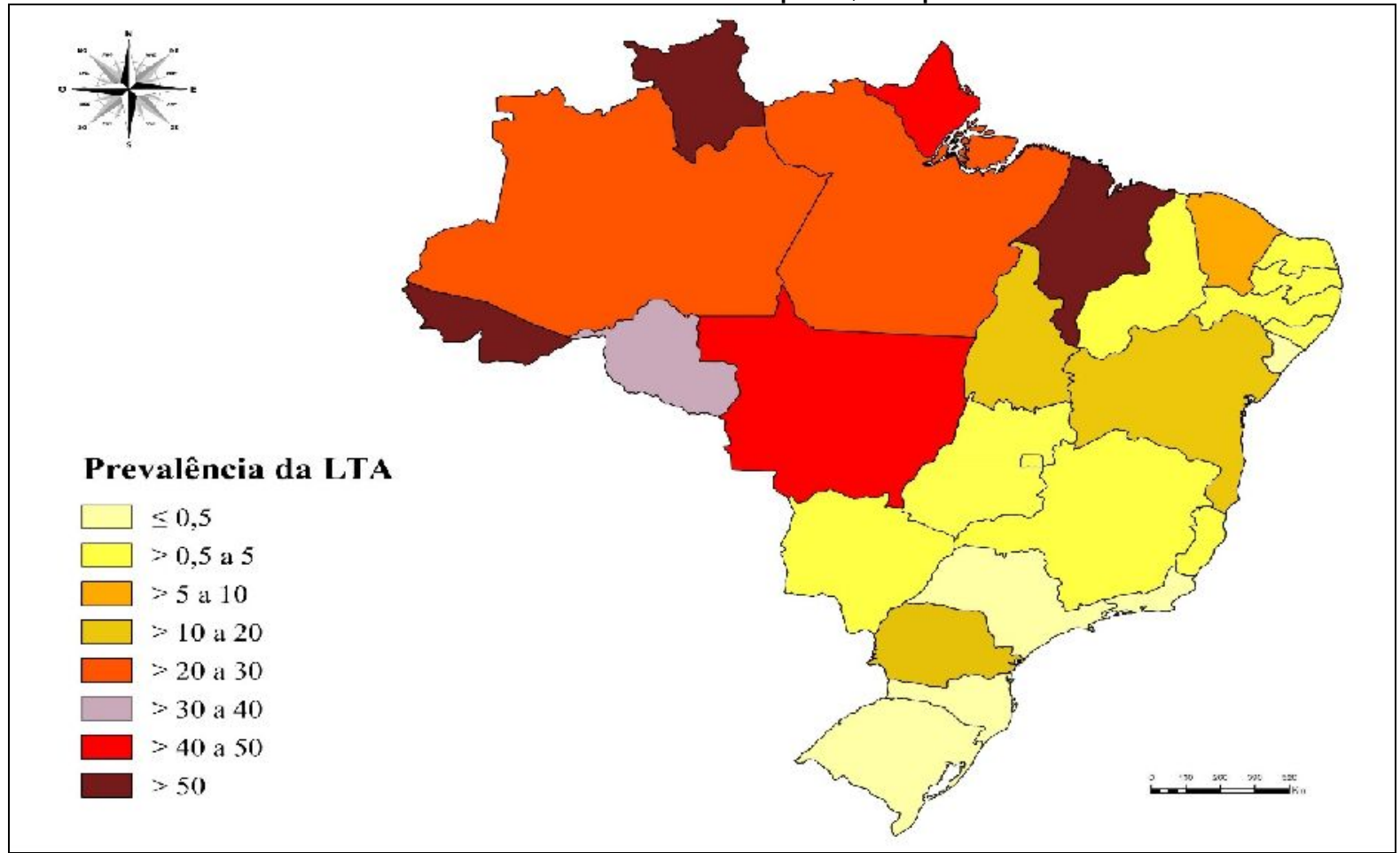

Fonte: Adaptado do SINAN (2013).

$\mathrm{Na}$ mesorregião do Agreste Pernambucano a LTA acometeu com maior frequência o sexo masculino, principalmente entre 20 a 39 anos de idade, e teve correlação positiva linear forte com a zona de residência rural e fraca com o índice de desenvolvimento humano e produto interno bruto per capita dos municípios estudados (Figura 4).

FIGURA 3 - Distribuição da LTA em pacientes humanos, no período de 2009 a 2013: (a) casos confirmados no Brasil, (b) prevalência de enfermidade no país, (c) casos confirmados e (d) prevalência em uma área endêmica do país (Estado de Pernambuco).

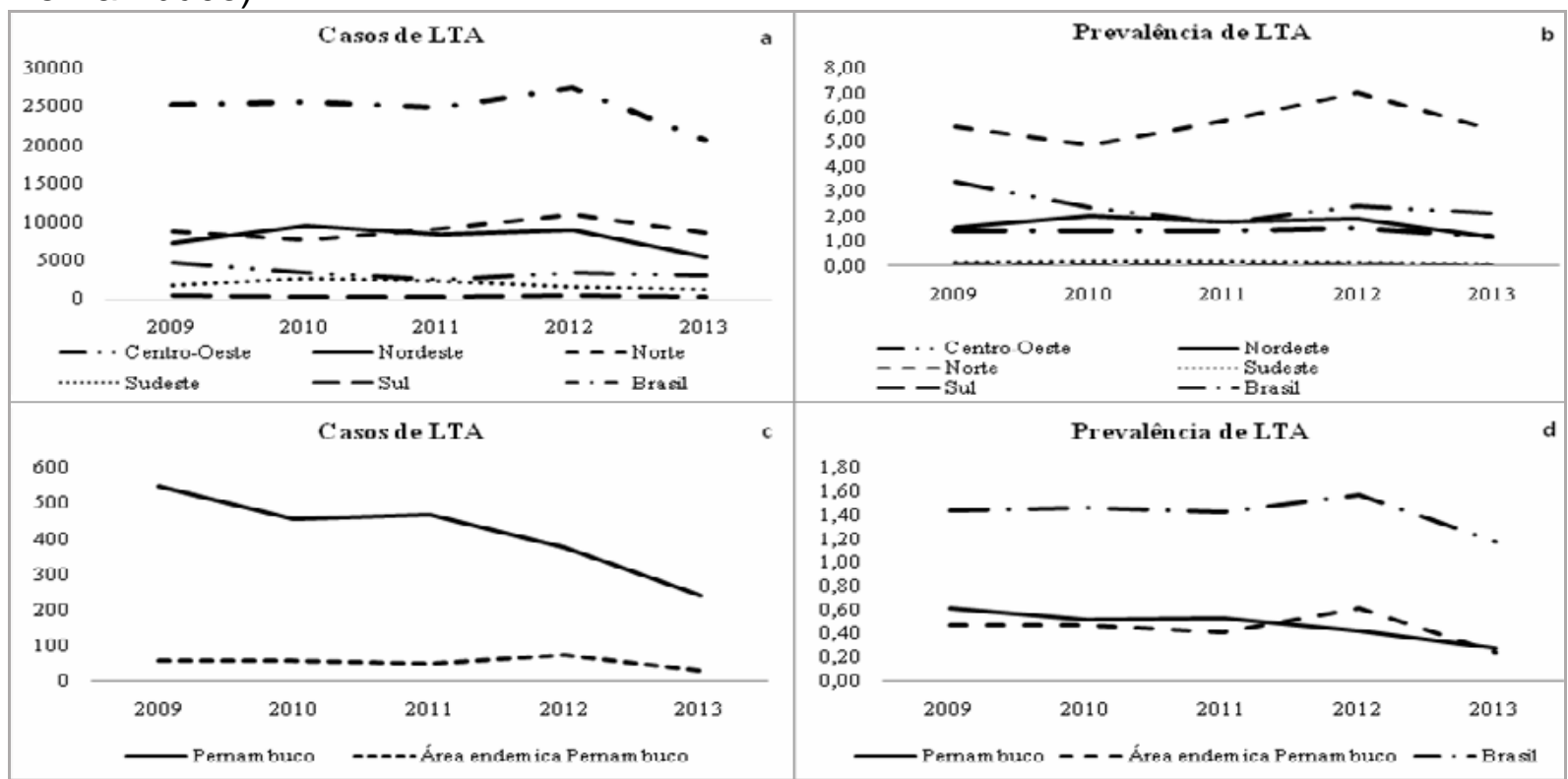

Fonte: Adaptado do SINAN (2013). 
FIGURA 4 - Avaliação da associação entre casos confirmados da enfermidade com índice de desenvolvimento humano (IDH), produto interno bruto (PIB) per capita dos municípios, e zona de residência urbana e rural, na área endêmica do Estado de Pernambuco, no período de 2009 a 2013.

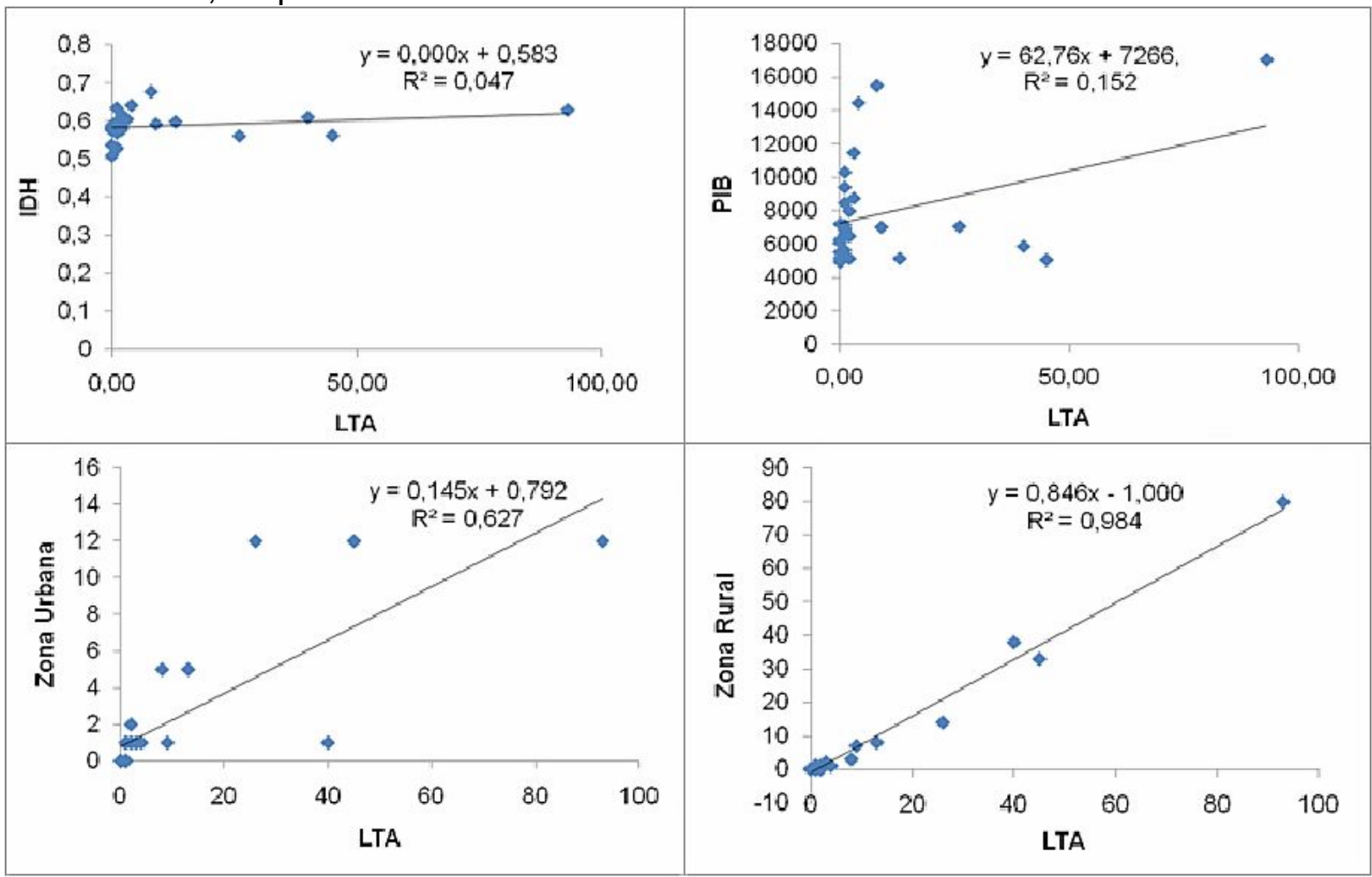

Fonte: Adaptado do SINAN (2013).

Nesse estudo a prevalência (6,49/10.000 hab) de casos da LTA em humanos foi superior as médias observadas em outros países, como no Paraguai $(1,66 /$ $10.000 \mathrm{hab})$ e Argentina ( $0,18 / 10.000 \mathrm{hab})$ segundo dados apresentados pela Pan American Health Organization - PAHO $(2013,2019)$, sendo justificável em virtude do tamanho superior da amostra populacional (sadia e doente) em relação ao grupo afetado considerado na avaliação.

Observou-se que pessoas residentes na região Norte, Centro-Oeste e Nordeste do Brasil foram as mais acometidas pela enfermidade, com predominância nos Estados situados na região da Amazônia Legal, como Acre, Amapá, Amazonas, Mato Grosso, Pará, Rondônia, Roraima, Tocantins e Maranhão. Em 2010 a taxa de incidência registrada na Amazônia foi de 70,26 casos confirmados a cada 100.000 habitantes, tendo revelado os maiores coeficientes os estados do Acre, Roraima, Mato Grosso, Amapá e Rondônia variando entre 59,97 a 141,07 casos a cada 100.000 habitantes (VIANA et al., 2016).

Nessa região há uma intensa taxa de desmatamento, que se estende desde o Maranhão até Rondônia (LEMOS; SILVA, 2011; SACCARO JÚNIOR et al., 2015), e contribui para mudanças climáticas que, de forma direta ou indireta, podem produzir impactos sobre a saúde humana por alterar ecossistemas e ciclos biogeoquímicos, favorecendo o aumento da incidência de doenças infecciosas como a LTA (BARCELLOS et al., 2009; MENDES et al., 2016; AMARO; COSTA, 2017). A presença de LTA no território nacional pode estar relacionada com a condição climática, uma vez que a enfermidade ocorre predominantemente em regiões tropicais e subtropicais (BRASIL, 2006). 
As diferentes proporções de LTA, por regiões geográficas e unidades federativas, encontradas no estudo relacionam-se com as especificidades dos ecossistemas associados aos fatores antrópicos, que favorecem ou não atores da cadeia epidemiológica da enfermidade. Algumas atividades antropogênicas, como o desmatamento e formação de ilhas de calor, ocasionam mudanças climáticas capazes de influenciar na distribuição e abundância dos padrões de plantas e animais, produzindo efeitos na dinâmica das doenças vetoriais (PBMC, 2014; MORAES et al., 2020), tais modificações acabam promovendo a migração dos vetores ou selecionando espécies com melhor capacidade adaptativa, possivelmente aquelas com menores necessidades alimentares e climáticas, podendo ainda possibilitar uma maior disseminação de animais silvestres, fontes de alimento para os flebotomíneos (SANTOS et al., 2005; FARIA et al., 2019; GOMES et al., 2015).

Já na região Norte a intensidade da prevalência de LTA pode estar relacionado ao ciclo silvestre de transmissão do protozoário. Estudos comprovam a participação de roedores, felídeos, canídeos, endentatus e primatas silvestres na cadeia epidemiológica da Leishmania sp. (RANGEL et al., 2018; ALEMAYEHU; ALEMAYEHU, 2017). É importante destacar que o principal vetor nessa região é o Lutzomyia umbratilis, o qual pode ser encontrado em países fronteiriços ao Brasil e também nos estados do Acre, Amapá, Amazonas, Maranhão, Mato Grosso, Pará, Roraima e Rondônia (BRASIL, 2010; FREITAS et al., 2015).

A região Centro-Oeste por ser considerada área de transição, devido a sua localização limitar-se ao norte, a região equatorial, a noroeste a região semiárida e ao sul a região subtropical, favorece a proliferação e manutenção dos vetores flebotomíneos (HIDASI FILHO; GARCIA-ZAPATA, 2004). Além de crescentes áreas de ocupações de interesse econômico para agricultura, pecuária e extrativismo de madeira e minerais que também podem favorecer o ciclo silvestre da LTA (DUARTE, 2003).

Observou-se que, dentre os Estados do Nordeste, os que apresentaram maior prevalência desta zoonose, Maranhão e Bahia, são aqueles que contêm maior diversidade de fauna de flebotomíneos (AGRA et al., 2016; REBÊLO et al., 2010). Nessa região, a LTA ocorre em dois padrões de transmissão, sendo um rural relacionado com o contato do ser humano com o ambiente silvestre, e outro urbano ou periurbano, onde os flebotomíneos são encontrados nas imediações e dentro dos domicílios, devido a sua grande adaptação a ecótopos artificiais (SILVA et al., 2010). Vale destacar, que no Maranhão, além da questão do desmatamento da Amazônia Legal, estudos indicam que dentre os estados nordestino é o que menor apresenta capacidade para enfrentar as duas formas da leishmaniose (PBMC, 2014).

Ainda na região Nordeste, o Estado de Pernambuco, também merece destaque em função da diversidade e ampla distribuição geográfica da fauna flebotomínea (BRITO et al., 2012; SILVA et al., 2017), a qual tem sido descrita em torno de 37 espécies pertinentes a 14 gêneros (DANTAS-TORRES et al., 2010).

Em Pernambuco no período de 2009 a 2013, a proporção de casos de LTA humana diminuiu, exceto em áreas endêmicas localizadas na mesorregião do Agreste Pernambucano, em municípios alocados na quarta Região Administrativa de Saúde Estadual, onde houve persistência da enfermidade e presença de pico epidêmico. Este pico ocorreu no período de grande transição climática na região, propiciando de forma direta ou indireta o contato de humanos com flebotomíneos e reservatórios silvestres ou sinantrópicos. Para Chagas et al. (2016) o número de vetores pode aumentar em decorrência do aumento da pluviosidade, pois, a 
umidade acaba favorecendo a eclosão das pupas, resultando em maior atividade fora dos seus abrigos (ALMEIDA et al., 2010).

Nessa região endêmica, alguns aspectos epidemiológicos avaliados assemelham-se ao que vem ocorrendo em outras áreas do Brasil, como a maior ocorrência da enfermidade em indivíduos do sexo masculino em idade produtiva e em habitantes de áreas de baixo índice de desenvolvimento humano, uma vez que a ocorrência da LTA em indivíduos do sexo masculino, jovens e adultos, em fase produtiva, caracteriza a ocorrência do perfil de transmissão ocupacional da enfermidade nas frentes de trabalho (BRASIL, 2010; GONÇALVES et al., 2019; RIBEIRO et al., 2020). Já em áreas de baixo índice de desenvolvimento humano, a ocorrência de LTA está relacionada com situação precária de saneamento básico e a situação socioeconômica, aliada a outros aspectos sanitários (ARAÚJO et al., 2016; MAIA et al., 2017; SANTI, 2017; RODRIGUES et al., 2018).

A correlação entre casos humanos e zona rural em áreas endêmicas localizadas na mesorregião do Agreste Pernambucano pode estar relacionada às práticas agrícolas e ao ecoturismo, sendo as principais atividades econômicas da região a agricultura, pecuária, e turismo rural (SOBEL et al., 2009). Destacando-se que os municípios mais procurados para prática de ecoturismo nessa região como os de Bezerros, Bonito, Gravatá, Taquaratinga do Norte, Brejo da Madre de Deus, Caruaru e São Caetano (DIAS; VITAL, 2014), representaram 35\% (93/261) de casos humanos da LTA.

A análise de situações particulares de detecção da LTA deve-se incluir novas metodologias que avaliem extensas séries temporais, eventos e áreas sentinela e utilizem ferramentas do geoprocessamento (PBMC, 2014; MOREIRA et al., 2016), de modo a fornecer melhores informações sobre a dinâmica das variáveis climáticoambientais envolvidas nos modelos integrados de caracterização de risco dessa zoonose (BARCELLOS et al., 2009).

\section{CONCLUSÃO}

A LTA em humanos ocorre em diferentes proporções nas unidades federativas do Brasil, sobretudo nas regiões Norte, Centro-oeste e Nordeste, devido às variações das condições climáticas no território nacional, que influenciam na taxa de reprodução dos flebotomíneos, potencializando assim a transmissão da enfermidade. As estratégicas de prevenção da LTA devem ser reavaliadas e incluir mecanismos que avaliem todos os aspectos envolvidos na cadeia epidemiológica, o que indica a necessidade do desenvolvimento de pesquisas que identifiquem as particularidades de todos os atores epidemiológicos em cada unidade federativa.

\section{REFERÊNCIAS}

AGRA, M. C. R.; COSTA, P. L.; DUQUE, A. E. S.; SOARES, E. N. L.; ALVES, L. C. et al. Sandflies (Diptera: Psychodidae) in an urban area of Northeastern Brazil. Revista da Sociedade Brasileira de Medicina Tropical, v. 49, n. 6, p. 698-702, Uberaba, 2016. Disponível em: <http://dx.doi.org/10.1590/0037-8682-0147-2016>. DOI: 10.1590/0037-8682-0147-2016

ALEMAYEHU, B.; ALEMAYEHU, M. Leishmaniasis: A Review on Parasite, Vector and Reservoir Host. Health Science Journal, v. 11, n. 4., p. 519, 2017. Disponível em: <http://dx.doi.org/10.21767/1791-809x.1000519>. DOI: 10.21767/1791809x.1000519 
ALMEIDA, P. S.; MINZÃO, E. R.; MINZÃO, L. D.; SILVA, S. R.; FERREIRA, A. D. et al. Aspectos ecológicos de flebotomíneos (Diptera: Psychodidae) em área urbana do Município de Ponta Porã, Estado de Mato Grosso do Sul. Revista da Sociedade Brasileira de Medicina Tropical, v. 43, n. 6, p. 723-727, Uberaba, 2010. Disponível em: <https://doi.org/10.1590/S0037-86822010000600025>. DOI: 10.1590/S003786822010000600025

AMARO, R. R.; COSTA, W. W. Transformações socioespaciais no estado do Rio de Janeiro enquanto determinante social da saúde: no contexto das leishmanioses. Hygeia - Revista Brasileira de Geografia Médica e da Saúde, v. 13, n. 26, p. 198 210, 2017. Disponível em: <http://dx.doi.org/10.14393/Hygeia132617>. DOI: 10.14393/Hygeia132617

ARAÚJO, A. R.; PORTELA, N. C.; FEITOSA, A. P. S.; SILVA, O. ALVES et al. Risk factors associated with American Cutaneous Leishmaniasis in an endemic area of Brazil. Revista do Instituto de Medicina Tropical de São Paulo, v. 58, p. 1-6, 2016. Disponível em: <http://dx.doi.org/10.1590/s1678-9946201658086>. DOI: $10.1590 /$ S1678-9946201658086

ARAUJO, L. M.; MAIA, D. C. C.; AGONIO; B.; CERDAN, L. M.; GALVÃO, R. S. Leishmaniose tegumentar americana - apresentação atípica diagnosticada com técnica de biologia molecular. Revista da Sociedade Portuguesa de Dermatologia e Venereologia (SPDV), v. 73, n. 4, 2015 . Disponível em: <http://dx.doi.org/10.29021/spdv.73.4.490>. DOI: 10.29021/spdv.73.4.490

BARCELLOS, C.; MONTEIRO, A. M. V.; CORVALAN, C.; CARVALHO, M. S.; HACON, S. et al. Mudanças climáticas e ambientais e as doenças infecciosas: cenários e incertezas para o Brasil. Revista Epidemiologia e Serviços de Saúde, v. 18, n. 3, p. 285-304, Brasília, 2009. Disponível em: <http://scielo.iec.gov.br/pdf/ess/v18n3/v18n3a11.pdf>. Acesso em: 05 Jun. 2020.

BRASIL, Ministério da Saúde. Manual de Vigilância da Leishmaniose Tegumentar Americana. Série A. Normas e Manuais Técnicos, 1. a edição 3. a reimpressão, Brasília - $\quad$ DF, 2006. Disponível em:< http://bvsms.saude.gov.br/bvs/publicacoes/manual_vigilancia_controle_leishmaniose _visceral.pdf>. Acesso em: 16 Jun. 2020.

BRASIL, Ministério da Saúde. Manual de Vigilância da Leishmaniose Tegumentar Americana. Série A. Normas e Manuais Técnicos, 2ª edição, Brasília - DF, 2007. Disponível em: http://bvsms.saude.gov.br/bvs/publicacoes/manual_vigilancia_leishmaniose_2ed.pdf >. Acesso em: 28 Ago. 2020.

BRASIL, Ministério da Saúde. Manual de Vigilância da Leishmaniose Tegumentar Americana. Série A. Normas e Manuais Técnicos, 2. a edição atualizada 1.. a reimpressão, Brasília $\quad$ - $\quad$ DF, 2010. Disponível em:<http://bvsms.saude.gov.br/bvs/publicacoes/manual_vigilancia_leishmaniose_teg umentar_americana.pdf>. Acesso em: 16 Jun. 2020. 
BRASIL, Ministério da Saúde. Distribuição da Leishmaniose Tegumentar (LT) no Brasil e mundo. 16 Ago. 2019. Disponível em: <https://saude.gov.br/saude-de-az/leishmaniose-tegumentar>. Acesso em: 28 Ago. 2020.

BRITO, M. E. F.; ANDRADE, M. S.; DANTAS-TORRES, F.; RODRIGUES, E.H.G.; CAVALCANTI, M.P. et al. Cutaneous Leishmaniasis in northeastern Brazil: a critical appraisal of studies conducted in State of Pernambuco. Revista da Sociedade Brasileira de Medicina Tropical, v. 45, n. 4, p. 425-429, Uberaba, 2012. Disponível em: <http://dx.doi.org/10.1590/S0037-86822012005000006>. DOI: 10.1590/S003786822012005000006

CAETANO, D. C. D. S.; OKUYAMA, C. E.; SANTOS, M. R. M.; PEREIRA, R. M. S. Parâmetros clínicos, diagnóstico e tratamento da Leishmaniose Tegumentar e visceral. Enciclopédia Biosfera, v. 16, n. 29, p. 2087-2105, Goiânia, 2019. Disponível em: <http://dx.doi.org/10.18677/EnciBio_2019A161>. DOI: 10.18677/EnciBio_2019A161

CHAGAS, A. P.; SOARES, D. C.; SOUSA, G. C. R.; VIANA, R. B.; REBELO, J. M. M. et al. Aspectos ecológicos da fauna de flebotomíneos em focos de leishmaniose na Amazônia Oriental, Estado do Pará, Brasil. Revista Pan-Amazônica de Saúde, v. 7 n.esp, Ananindeua, 2016. Disponível em: <http://dx.doi.org/10.5123/s217662232016000500014>. DOI: 10.5123/s2176-62232016000500014

CURTI, M. C. M.; SILVEIRA, T. G. V.; ARRAES, S. M. A. A.; BERTOLINI, D. A.; ZANZARINI, P. D. et al. Aspectos epidemiológicos da Leishmaniose Tegumentar Americana na região Noroeste do Estado do Paraná. Revista de ciências farmacêuticas básica e aplicada, v. 30, n. 1, p. 63-68, 2009. Disponível em: $<$ http://files.bvs.br/upload/S/1808-4532/2009/v30n1/a005.pdf>. Acesso em: 05 Jun. 2020.

DANTAS-TORRES, F. D.; ANDRADE, A. J.; TENÓRIO, K. E. R.; ANDRADE FILHO, J. D.; BALBINO, V. Q. et al. Flebotomíneos, moscas areia, (Diptera: Psychodidae: Phlebotominae), no estado de Pernambuco. Revista da Sociedade Brasileira de Medicina Tropical, v. 43, n. 6, Uberaba, 2010. Disponível em: $<$ http://dx.doi.org/10.1590/S0037-86822010000600028>. DOI: $10.1590 / S 0037-$ 86822010000600028

DANTAS-TORRES, F.; SALES, K. G. S.; SILVA, L. G.; OTRANTO, D.; FIGUEREDO, L. A. Leishmania-FAST15: A rapid, sensitive and low-cost real-time PCR assay for the detection of Leishmania infantum and Leishmania braziliensis kinetoplast DNA in canine blood samples. Molecular and Cellular Probes, v. 31, p. 65-69, 2017. Disponível em: $\quad$ http://dx.doi.org/10.1016/j.mcp.2016.08.006>. DOI: 10.1016/j.mcp.2016.08.006

DIAS, P. P.; VITAL, T. W. O Ecoturismo no Estado de Pernambuco: uma visão do segmento a partir da oferta de serviços. Revista Turismo em Análise, v. 25, n. 2, p. 316-336, 2014. Disponível em: <http://dx.doi.org/10.11606/issn.19844867.v25i2p316-336>. DOI: 10.11606/issn.1984-4867.v25i2p316-336

DUARTE, J. L. S. Leishmaniose Tegumentar Americana no estado de Mato Grosso - 2002. Revista da Sociedade Brasileira de Medicina Tropical, Uberaba, v. 
36, supl. 2, 2003. Disponível em: <http://dx.doi.org/10.1590/S003786822003000700015>. DOI: 10.1590/S0037-86822003000700015

FARIA, H. G.; MAGNAGO, L. R. F.; JESUS, G. S.; ALMEIDA, Y. V.; POLEZE, L. S. et al. Pesquisa de Leishmania spp. em flebotomíneos (Diptera: Psychodidae) do munícipio de lúna, Espírito Santo. PUBVET, v. 13, n. 6, a 342, p.1-8, 2019. Disponível em: <http://dx.doi.org/10.31533/pubvet.v13n6a342.1-7>. DOI: 10.31533/pubvet.v13n6a342.1-7

FERNANDES, A. C. B. S.; PEDROSO, R. B.; VENAZZI, E. A. S.; ZANZARINI, P. D.; ARISTIDES, S. M. A. et al. American Cutaneous Leishmaniasis with unusual clinical presentation and response to treatment. Revista do Instituto de Medicina Tropical de São Paulo, v. 58, n. 0, 2016. Disponível em: <http://dx.doi.org/10.1590/S16789946201658020 >. DOI: 10.1590 / s1678-9946201658020

FERREIRA, L. T.; GOMES, A. H. S.; PEREIRA-CHIOCCOLA, V. L. Genotype characterization of Leishmania (Viannia) braziliensis isolated from human and canine biopsies with American Cutaneous Leishmaniasis. Revista do Instituto de Medicina Tropical, v. 57, n. 3, p. 257 - 262, São Paulo, 2015. Disponível em: <http://dx.doi.org/10.1590/S0036-46652015000300013>. DOI: $10.1590 /$ S0036-46652015000300013

FREITAS, M. T. S.; RÍOS-VELASQUES, C. M.; COSTA Jr, C. R. L. C; FIGUERÊDO $\mathrm{Jr}$, C. A. S.; ARAGÃO, N. C. et al. Phenotypic and genotypic variations among three allopatric populations of Lutzomyia umbratilis, main vector of Leishmania guyanensis. Parasites Vectors, v. 8, n. 1, p. 448, 2015. Disponível em: <https://dx.doi.org/10.1186/s13071-015-1051-7>. DOI: 10.1186/s13071-015-1051-7

GALGAMUWA, L. S.; DHARMARATNE, S. D.; IDDAWELA, D. Leishmaniose no Sri Lanka: distribuição espacial e variações sazonais de 2009 a 2016. Parasites Vectors, v. 11, n. 60, 2018. Disponível em: <http://dx.doi.org/10.1186/s13071-0182647-5>. DOI: $10.1186 / \mathrm{s} 13071-018-2647-5$

GOMES, J. S.; OLIVEIRA, F. S.; CARDOZO, S. V.; PACHECO, R. S. Importância da técnica de reação em cadeia da polimerase (PCR) no diagnóstico específico de leishmaniose tegumentar americana. Revista UNIABEU, v. 8, n. 20, 2015. Disponível em: <https://revista.uniabeu.edu.br/index.php/RU/article/view/2067>. Acesso em: 28 Ago. 2020.

GONÇALVES, N. V.; MIRANDA, C. S. C.; COSTA, R. J. F; GUEDES, J. A.; MATSUMURA, E. S. S. et al. Cutaneous Leishmaniasis: Spatial distribution and environmental risk factors in the state of Pará, Brazilian Eastern Amazon. The Journal of Infection in Developing Countries, v. 13, n. 10, p. 939-944, 2019. Disponível em: <https://doi.org/10.3855/jidc.11573>. DOI: 10.3855/jidc.11573

GONTIJO B.; CARVALHO, M. L. R. Leishmaniose tegumentar americana. Revista da Sociedade Brasileira de Medicina Tropical, v. 36, n. 1, p. 71-80, 2003. Disponível em: <http://dx.doi.org/10.1590/S0037-86822003000100011>. DOI: $10.1590 /$ S0037-86822003000100011 
HIDASI FILHO; GARCIA-ZAPATA, M. T. Correlação das Leishmanioses humanas com o bioma cerrado no Estado de Goiás. Universitas: Ciências da Saúde, v. 2, n. 1, p. 1-151, Brasília, 2004. Disponível em: <http://dx.doi.org/10.5102/UCS.V2l1.527>. DOI: 10.5102/UCS.V2I1.527

LEMOS, A. L. F.; SILVA, J. A. Desmatamento na Amazônia Legal: Evolução, Causas, Monitoramento e Possibilidades de Mitigação Através do Fundo Amazônia. Floresta e Ambiente, v. 18, n. 1, p. 98-108, 2011. Disponível em: < http://dx.doi.org/10.4322/floram.2011.027>. DOI: 10.4322/floram.2011.027

MAIA, J. A.; MENEZES, F. A.; SILVA, R. L.; SILVA, P. J. C. Características sociodemográficas de pacientes com Leishmaniose Tegumentar Americana. Revista Enfermagem Contemporânea, v. 6, n. 2, p.114-121, 2017. Disponível em: <http://dx.doi.org/10.17267/2317-3378rec.v6i2.1340>. DOI: 10.17267/23173378rec.v6i2.1340

MENDES, C. S.; COELHO, A. B.; FÉRES, J. G.; SOUZA, E. C.; CUNHA, D. A. Impacto das mudanças climáticas sobre a leishmaniose no Brasil. Revista Ciência \& Saúde Coletiva, vol.21 no.1 Rio de Janeiro jan. 2016. Disponível em: <http://dx.doi.org/10.1590/1413-81232015211.03992015>. DOI: $10.1590 / 1413-$ 81232015211.03992015

MORAES, J. L. P.; SANTANA, H. T. M.; BANDEIRA, M. C. A.; REBÊLO, J. M. M. Effects of forest degradation on the sand fly communities of northeast Brazil. Journal of Vector Ecology, v. 45, n. 1, p. 89-99, 2020. Disponível em: <http://dx.doi.org/10.1111/jvec.12376 DOI: 10.1111/ jvec.12376

MOREIRA, C.; SEGUNDO, A. S.; CARVALHOSA, A. A.; ESTEVAM, L. S.; PEREIRA, S. et al. Comportamento Geoespacial da Leishmaniose Tegumentar Americana no Município de Tangará da Serra - MT. Journal of Health Sciences, v. 18, n. 3, p. 171- 176, 2016. Disponível em: <http://dx.doi.org/10.17921/24478938.2016v18n3p171-6>. DOI: 10.17921/2447-8938.2016v18n3p171-6

NEGRÃO, G. N.; FERREIRA, M. E. M. C. Considerações sobre a dispersão da Leishmaniose Tegumentar Americana nas Américas. Revista Percurso (Online), v. 1 n. $\quad 1, \quad$ p. $85-103, \quad 2009 . \quad$ Disponível em: <http://periodicos.uem.br/ojs/index.php/Percurso/article/view/49453/751375140509>. Acesso em: 16 Jun. 2020.

NEGRÃO, G. N.; FERREIRA, M. E. M. C. Considerações sobre a leishmaniose tegumentar americana e sua expansão no território brasileiro. Revista Percurso NEMO, v. 6, n. 1, p. 147- 168, Maringá, 2014. Disponível em: <http://dx.doi.org/10.4025/revpercurso.v6i1.21375>. $10.4025 /$ revpercurso.v6i1.21375

OMS, Organização Mundial de Saúde . Leishmaniasis. 2 mar. 2020. Disponível em: <https://www.who.int/es/news-room/fact-sheets/detail/leishmaniasis>. Acesso em: 15 Jun. 2020. 
PAHO, Pan American Health Organization. Leishmaniasis. Informe epidemiológico de las Américas. Abril, 2013 Disponível em: <https://iris.paho.org/handle/10665.2/51678>. Acesso em: 18 Jun. 2020.

PAHO, Pan American Health Organization. Leishmaniasis. Epidemiological Report of the Americas. December, 2019. Disponível em: <https://iris.paho.org/handle/10665.2/51738>. Acesso em: 18 Jun. 2020.

PBMC, Painel Brasileiro de Mudanças Climáticas. Impactos, vulnerabilidades e adaptação às mudanças climáticas. Contribuição do Grupo de Trabalho 2 do Painel Brasileiro de Mudanças Climáticas ao Primeiro Relatório da Avaliação Nacional sobre Mudanças Climáticas [Assad, E.D., Magalhães, A. R. (eds.)]. COPPE. Universidade Federal do Rio de Janeiro, Rio de Janeiro, RJ, Brasil, 414 pp., 2014. Disponível em: <http://www.pbmc.coppe.ufrj.br/documentos/RAN1_completo_vol2.pdf>. Acesso em: 05 Jun. 2020.

RANGEL, E. F.; LAINSON, R.; CARVALHO, B. M.; COSTA, S. M.; SHAW, J. J. Sand fly Vectors of American Cutaneous Leishmaniasis in Brazil. In: RANGEL, E. F.; SHAW, J. J. Brazilian sand flies: biology, taxonomy, medical importance and control. Gewerbestrasse: Springer, p. 340-380, 2018. Disponível em: <http://dx.doi.org/10.1007/978-3-319-75544-1_7>. DOI: 10.1007/978-3-319-755441 1 7

REBÊLO, J. M. M.; ROCHA, R. V.; MORAES, J. L. P.; SILVA, C. R. M.; LEONARDO, F. S. et al. The fauna of phlebotomines (Diptera, Psychodidae) in different phytogeographic regions of the state of Maranhão, Brazil. Revista Brasileira de Entomologia, v. 54, n. 3, 2010. Disponível em: <http://dx.doi.org/10.1590/S008556262010000300022>. DOI: 10.1590/S0085-56262010000300022

RIBEIRO, A. C. S.; SANTANA, D. A.; SILVA, S. G.; RABELO, L. M.; ALEXANDRE, K. V. et al. Profilaxias da Leishmaniose Tegumentar Americana: papel do enfermeiro frente a enfermidade. Revista Liberum Accessum v. 1, n. 1, 2020. Disponível em: $<$ http://revista.liberumaccesum.com.br/index.php/RLA/article/view/15/13>. Acesso em: 15 Jun. 2020.

RODRIGUES, M. G. A.; SOUSA, J. D. B.; DIAS, Á. L. B.; MONTEIRO, W. M.; SAMPAIO, V. S. The role of deforestation on American Cutaneous Leishmaniasis incidence: spatial-temporal distribution, environmental and socioeconomic factors associated in the Brazilian Amazon. Tropical Medicine \& International Health, v. 24, n. 3, 2018. Disponível em: <http://dx.doi.org/10.1111/tmi.13196>. DOI:10.1111/tmi.13196

SACCARO JÚNIOR, N. L.; MATION, L. F.; SAKOWSKI, P. A. M. Impacto do desmatamento sobre a incidência de doenças na Amazônia. Texto para discussão - Instituto de Pesquisa Econômica Aplicada. Brasília: Rio de Janeiro: IPEA, 2015. Disponível em:

https://www.researchgate.net/profile/Nilo_Saccaro_Jr/publication/283503762_IMPAC TO_DO_DESMATAMENTO_SOBRE_A_INCIDENCIA_DE_DOENCAS_NA_AMAZO NIA/links/563b997c08ae405111a76efb. pdf>. Acesso em: 17 Jun. 2020. 
SANTI, A. C. Úlceras por leishmaniasis. Revista Flebología, v. 43, n. 1, 2017. Disponível em: <http://www.sociedadflebologia.com/Revistas/2017/Vol-43n1/Vol43N1-PDF14.pdf>. Acesso em: 17 Jun. 2020.

SANTOS, G. P. L.; SANAVRIA, A.; MARZOCHI, M. C. A.; SANTOS, E. G. O. B.; SILVA, V. L. et al. Prevalência da infecção canina em áreas endêmicas de leishmaniose tegumentar americana, do município de Paracambi, Estado do Rio de Janeiro, no período entre 1992 e 1993. Revista da Sociedade Brasileira de Medicina Tropical, v. 38, n. 2, p. 161-166, 2005. Disponível em: <https://www.arca.fiocruz.br/handle/icict/37818>. Acesso em: 28 Ago. 2020.

SANTOS, T. V.; PRÉVOT, G.; GINOUVÈS, M.; DUARTE, R.; SILVEIRA, F. T. et al. Ecological aspects of Phlebotomines (Diptera: Psychodidae) and the transmission of AMERICAN CUTANEOUS LEISHMANIASIS agents in an Amazonian/ Guianan bordering area. Parasites \& Vectors, v. 11, p. 612, 2018. Disponível em: <http://dx.doi.org/10.1186/s13071-018-3190-0>. DOl: 10.1186/s13071-018-3190-0

SHIMABUKURO, P. H. F.; GALATI, E. A. B. Lista de espécies de Phlebotominae (Diptera, Psychodidae) do Estado de São Paulo, Brasil, com comentários sobre sua distribuição geográfica. Biota Neotropica, vol. 11, supl. 1, 685-704, 2011. Disponível em: <https://dx.doi.org/10.1590/S1676-06032011000500033>. DOI: 10.1590/s1676-06032011000500033

SILVA, A. P. O.; MIRANDA, D. E. O.; SANTOS, M. A. B.; GUERRA, N. R.; MARQUES, S. R. et al. Phlebotomines in an area endemic for American Cutaneous Leishmaniasis in Northeastern coast of Brazil. Brazilian Journal of Veterinary Parasitology, Jaboticabal, v. 26, n. 3, p. 280-284, 2017 Disponível em: <http://dx.doi.org/10.1590/S1984-29612017038>. DOI: 10.1590/S1984-29612017038

SILVA, T. C.; GOMES, L. M. P. R.; PEREIRA, Y. N. O.; MORENO, M. C.; REBÊLO, J. M. M. Perfil epidemiológico da Leishmaniose Tegumentar Americana (LTA) em municípios do interior do estado do Maranhão, Brasil. Revista Saúde, v .4, (Esp. 1), p. 88, 2010. Disponível em: <http://revistas.ung.br/index.php/saude/article/download/665/754>. Acesso em: 18 Jun. 2020.

SINAN, Sistema de Informação de Agravos de Notificação. Dados Epidemiológicos Sinan. Disponível em: <https://portalsinan.saude.gov.br/dados-epidemiologicossinan>. Acesso em: 15 Mai. 2020.

SOBEL, T. F.; MUNIZ, A. L. P.; COSTA, E. F. Divisão regional do desenvolvimento humano em Pernambuco: uma aplicação da análise de cluster. Teoria e Evidência Econômica, v. 15, n. 33, p. 37-62, 2009. Disponível em: <http://dx.doi.org/10.5335/rtee.v15i33.4272>. DOI: 10.5335/rtee.v15i33.4272

VASCONCELOS, J. M.; GOMES, C. G.; SOUSA, A.; TEIXEIRA, A. B.; LIMA, J. M. Leishmaniose Tegumentar Americana: perfil epidemiológico, diagnóstico e tratamento. Revista Brasileira de Análises Clínicas, v. 50, n. 3, p. 221-227, 2018. 
Disponível em: $\quad$ <http://dx.doi.org/10.21877/2448-3877.201800722>. DOl: $10.21877 / 2448-3877.201800722$

VIANA, R. L.; FREITAS, C. M.; GIATTI, L. L. Saúde ambiental e desenvolvimento na Amazônia legal: indicadores socioeconômicos, ambientais e sanitários, desafios e perspectivas. Saúde e Sociedade. v. 25, n.1, p. 233-246., 2016. Disponível em: <http://dx.doi.org/10.1590/S0104-12902016140843>. DOI: 10.1590/S010412902016140843 